\title{
Estudo transversal da evolução da dentição decídua: forma dos arcos, sobressaliência e sobremordida ${ }^{\dagger}$
}

\section{Cross-sectional study of the evolution of the primary dentition: shape of dental arches, overjet and overbite}

\author{
Liana Amado de Castro* \\ Adriana Modesto** \\ Roberto Vianna** \\ Vera Lígia Mendes Soviero****
}

\begin{abstract}
RESUMO: O objetivo deste trabalho foi avaliar as características da dentição decídua de crianças de 6 a 39 meses de idade, matriculadas em quatro creches do município do Rio de Janeiro, Brasil. Foram avaliadas a forma dos arcos dentários, sobressaliência e sobremordida, relacionando-as com gênero e fase de desenvolvimento da dentição decídua. Com relação ao arco superior, 68,6\% das crianças possuíam arco arredondado e 31,4\% forma triangular. Quanto ao arco inferior, $92 \%$ o apresentaram em forma de "U" e $8 \%$ em forma de quadrado. A sobressaliência moderada ocorreu em $38,3 \%$, enquanto que a leve ocorreu em 30,3\% das crianças. A sobremordida exagerada (26,6\%) e a negativa (25,5\%) foram as mais prevalentes. Não houve diferença significante entre as freqüências das diferentes formas dos arcos dentários, sobressaliência e sobremordida em relação ao gênero. Houve uma associação estatisticamente significante (qui-quadrado, $\mathrm{p}<0,001$ ) entre a forma dos arcos superiores, o grau de sobressaliência e o grau de sobremordida em relação à fase de desenvolvimento da dentição. No presente estudo, foi possivel observar um aumento significativo das maloclusões com a erupção dos primeiros molares decíduos e elevação da dimensão posterior. Dessa forma, torna-se extremamente importante que a primeira visita odontológica ocorra no primeiro ano de vida, possibilitando ao odontopediatra a prevenção ou diagnóstico precoce de maloclusões na dentição decídua em desenvolvimento.

DESCRITORES: Dentição primária; Arcada dentária; Maloclusão; Pré-escolar; Prevalência.
\end{abstract}

\begin{abstract}
The aim of this study was to evaluate the characteristics (shape of dental arches, overjet and overbite) of the primary dentition of 6- to 39-month-old children from four nurseries of Rio de Janeiro, Brazil, and to associate them to gender and dentition phase. It was observed that $68.6 \%$ of the children presented round upper arch, while $31.4 \%$ had triangular upper arch; $92 \%$ of the children presented U-shaped lower arch and $8 \%$ had square-shaped lower arch. Moderate overjet was observed in 38.3\% of the children, and slight overjet, in 30.3\%. Severe (26.6\%) and negative $(25.5 \%)$ overbites were the most prevalent modalities of that condition. There was no association between gender and the studied characteristics. There was statistically significant association between the dentition phase and the shape of the arch, overjet and overbite ( $p<0.001$, chi-square test). It was possible to observe, in the present study, that early signs of malocclusions appeared when first primary molars erupted, as the posterior vertical dimension of occlusion increased. It was, thus, verified that early signs of malocclusions appear as the primary dentition develops. It is very important that the first dental visit occur during the first year of age, since it allows the pediatric dentist to prevent or carry out an early diagnosis of malocclusions in the primary dentition.
\end{abstract}

DESCRIPTORS: Dentition, primary; Dental arch; Malocclusion; Child, preschool; Prevalence.

\section{INTRODUÇÃO}

Desde a sua formação, os arcos dentários fazem parte dos elementos básicos necessários para o estabelecimento do crescimento e desenvolvimento da face ${ }^{1,15}$. Ao nascer, a criança apresenta uma proporção da face em relação ao crânio correspondente a um quarto do tamanho total da cabeça ${ }^{4}$. Dois fatores são de grande importância nesta época. O primeiro é que praticamente todo o crescimento dos arcos dentários e da face ocorre após o nascimento da criança, e o segundo é que o sentido de crescimento da face é direcionado para baixo e para frente em relação à base do crânio ${ }^{15,16}$.

A observação da forma dos arcos dentários no período pós-natal é fundamental, uma vez que pode ocorrer um grande número de alterações re-

\footnotetext{
$\dagger$ Parte de Dissertação de Mestrado em Odontopediatria da Faculdade de Odontologia da Universidade Federal do Rio de Janeiro. *Mestranda; **Professores Adjuntos - Departamento de Odontopediatria e Ortodontia da Faculdade de Odontologia da Universidade Federal do Rio de Janeiro.

*** Professora Adjunta da Disciplina de Odontopediatria da Universidade do Estado do Rio de Janeiro e da Universidade Estácio de Sá.
} 
Castro LA de, Modesto A, Vianna R, Soviero VLM. Estudo transversal da evolução da dentição decídua: forma dos arcos, sobressaliência e sobremordida. Pesqui Odontol Bras 2002;16(4):367-373.

lacionadas com o desenvolvimento da face. Porém, devido à dificuldade no manejo da criança nesta faixa etária e ao seu pouco acesso ao atendimento odontológico, este grupo etário torna-se pouco explorado $^{9,17,20}$.

O fator genético é considerado preponderante no padrão de crescimento e desenvolvimento dos arcos dentários, porém este pode ser também significantemente influenciado pelos fatores ambientais, funções bucais, qualidade nutricional e saúde do indivíduo ${ }^{7,16,20}$. Este pensamento é consubstanciado pela teoria da matriz funcional de Moss $^{12,13,14}$ (1997), segundo a qual o crescimento da face ocorre em resposta às necessidades funcionais, o que significa conceitualmente que os tecidos moles crescem e os ossos e cartilagens respondem a este estímulo ${ }^{12,13,14}$.

A forma dos arcos dentários é fortemente influenciada pelas funções bucais e pelo crescimento vertical dos processos alveolares, em resposta ao estímulo da erupção dos dentes ${ }^{15,16}$. Portanto, no que diz respeito à oclusão na dentição decídua, os fatores funcionais são considerados relevantes na etiologia das maloclusões em pacientes saudáveis $^{1,7,16,20}$.

A relação horizontal e vertical entre os arcos dentários pode ser influenciada pelos comportamentos culturais, como os hábitos alimentares (aleitamento natural e artificial) e hábitos bucais não-nutritivos (uso prolongado da mamadeira, sucção de chupeta e dedo) ${ }^{3,4,5,8,10,18}$.

Na população brasileira, os estudos sobre prevalência das maloclusões na dentição decídua revelam um padrão semelhante ao de alguns países industrializados (Quadro 1).

A forma dos arcos dentários, a sobressaliência e a sobremordida são medidas importantes para a avaliação da oclusão na dentição decídua em evolução. A sua observação é necessária na prevenção dos desvios oclusais que podem levar ao surgimen- to de maloclusões em idades bastante jovens. O objetivo deste trabalho foi verificar as características dos arcos dentários de crianças na faixa etária de 6 a 39 meses, o que abrange o início e o final da erupção dentária decídua. Os aspectos observados foram a forma dos arcos dentários e suas relações no sentido horizontal e vertical, correlacionando-os com gênero e fase de desenvolvimento da dentição decídua.

\section{MATERIAL E MÉTODO Tipo de estudo e amostra}

Este estudo foi do tipo transversal cuja amostra foi constituída de 188 crianças, saudáveis, de ambos os sexos, na faixa etária de 6 a 39 meses de idade, matriculadas em quatro creches, duas públicas e duas particulares do município do Rio de Janeiro - RJ, Brasil.

Este estudo foi aprovado pelo Comitê de Pesquisa em Humanos do Núcleo de Saúde Coletiva (NESC) da UFRJ-RJ e foi obtido por escrito, antes do início do estudo, o consentimento livre e esclarecido dos pais ou responsáveis.

\section{Critérios para o exame clínico}

Os exames clínicos foram realizados por um único examinador (L.A.C.), devidamente calibrado, auxiliado por um anotador, sob luz natural e através de inspeção visual, seguindo as normas de biosegurança. O examinador posicionava-se de frente para a criança que estava sentada em uma cadeira tipo bebê-conforto (6-14 meses) ou em uma pequena cadeira com a cabeça apoiada (15-39 meses). A amostra foi dividida em 4 grupos de acordo com a seqüência de erupção dos dentes decíduos: Grupo 0 (ausência de dentes), Grupo 1 (incisivos centrais ou laterais irrompidos), Grupo 2 (primeiros molares irrompidos, com ou sem caninos), Grupo 3 (dentição decídua completa).

QUADRO 1 - Estudos brasileiros sobre prevalência de sobressaliência, sobremordida e mordida aberta anterior.

\begin{tabular}{|c|c|c|c|c|c|c||}
\hline Autores & Ano & Faixa etária & Amostra (n) & $\begin{array}{c}\text { Sobressaliência } \\
\text { exagerada }\end{array}$ & $\begin{array}{c}\text { Sobremordida } \\
\text { exagerada }\end{array}$ & $\begin{array}{c}\text { Mordida aberta } \\
\text { anterior }\end{array}$ \\
\hline Valente, Mussolino $^{21}$ & 1989 & 2 a 6 anos & 120 & $10,83 \%$ & $10,83 \%$ & $23,32 \%$ \\
\hline Serra-Negra et al. ${ }^{18}$ & 1997 & 3 a 5 anos & 357 & $\begin{array}{c}9,3 \% \mathrm{~s} / \mathrm{HB} * \\
15,6 \% \mathrm{c} / \mathrm{HB} *\end{array}$ & $\begin{array}{c}52,5 \% \mathrm{~s} / \mathrm{HB} * \\
20,2 \% \mathrm{c} / \mathrm{HB}\end{array}$ & $31,9 \% \mathrm{c} / \mathrm{HB} *$ \\
\hline Soviero $^{19}$ & 1999 & 2 a 6 anos & 214 & $33,7 \%$ & $9,3 \%$ & $44,4 \%$ \\
\hline Tollara $^{20}$ & 2001 & 6 a 35 meses & 413 & $>50 \%$ & $20 \%-36,1 \%$ & $2,7 \%-38,5 \%$ \\
\hline
\end{tabular}

*Hábitos bucais. 
Castro LA de, Modesto A, Vianna R, Soviero VLM. Estudo transversal da evolução da dentição decídua: forma dos arcos, sobressaliência e sobremordida. Pesqui Odontol Bras 2002;16(4):367-373.

\section{Arcos dentários}

A forma dos arcos dentários superiores foi classificada em arredondada ou triangular e, dos inferiores, em forma de "U" ou quadrada. Segundo Camargo $^{4}$ (1998) e Villena, Correa ${ }^{22}$ (1998), o arco superior normal é arredondado, enquanto o inferior tem a forma de "U".

\section{Sobressaliência}

Medida com régua milimetrada de plástico, adaptada ao tamanho da cavidade bucal dos bebês, em oclusão cêntrica. Para as crianças do Grupo 0, esta avaliação foi realizada através da medição da distância entre os rodetes gengivais superior e inferior ${ }^{17}$. Para as crianças dos Grupos 1,2 e 3, mediu-se a distância entre os incisivos superiores e inferiores e a classificação utilizada foi ${ }^{15,16,21}$ :

- topo-a-topo - quando as bordas incisais dos incisivos superiores contactavam com as dos incisivos inferiores;

- leve - sobressaliência positiva não excedendo $2 \mathrm{~mm}$;

- moderada - sobressaliência positiva de 2,1 a $4 \mathrm{~mm}$;

- exagerada - sobressaliência positiva incisal acima de $4 \mathrm{~mm}$;

- negativa - mordida cruzada anterior.

\section{Sobremordida}

$\mathrm{Na}$ avaliação do grau de sobremordida, foram excluídas da amostra as crianças que não possuíam todos os incisivos centrais erupcionados.

Para as crianças do Grupo 0, esta avaliação foi realizada através da observação do trespasse vertical existente entre os rodetes gengivais superior e inferior que foram classificados segundo Sanche $z^{17}$ (2000). A partir do Grupo 1 foram utilizados os seguintes critérios para avaliação da sobremordida em oclusão cêntrica através do exame visual:

- topo-a-topo - as bordas dos incisivos superiores e inferiores se tocavam em um plano vertical;

- leve - o trespasse vertical dos incisivos superiores limitava-se ao terço incisal dos inferiores;

- moderada - o trespasse vertical atingia o terço médio dos incisivos inferiores;

- exagerada - o trespasse vertical dos incisivos superiores atingia o terço cervical dos incisivos inferiores;

- negativa - não havia trespasse vertical entre os incisivos superiores e inferiores, o que caracterizava a mordida aberta anterior.

\section{Análise estatística}

Os dados foram tabulados no programa estatístico Epi Info 6.04, utilizando o teste estatístico qui-quadrado, com nível de significância de 0,05.

\section{RESULTADOS}

A média de idade das 188 crianças examinadas foi de $23 \pm 9$ meses, sendo uniforme a distribuição com relação ao gênero: 101 meninos (53,7\%) e 87 meninas (46,3\%). Não houve diferença estatística das diferentes formas de arcos dentários, da sobressaliência e da sobremordida em relação ao gênero da criança $(p>0,05)$. Portanto, com relação às características bucais observadas neste estudo, o gênero constituiu uma variável não associada.

Houve uma maior prevalência dos arcos superiores com forma arredondada (129 - 68,6\%) e dos arcos inferiores em forma de "U" (173 - 92\%) (Tabela 1). A avaliação da forma dos arcos em relação à fase de desenvolvimento da dentição decídua demonstrou que, nas fases mais avançadas, a prevalência do arco superior triangular foi significativamente mais alta ( $\mathrm{p}<0,001)$. Entretanto, não foi observada diferença em relação às diferentes formas dos arcos dentários inferiores ( $p>0,05)$ (Tabela 2).

A freqüência da sobressaliência mais observada neste estudo foi a moderada $(72-38,3 \%)$, seguida da leve $(57-30,3 \%)$ (Tabela 3$)$. Apenas 4 $(2,1 \%)$ crianças apresentaram sobressaliência negativa e foram excluídas das análises de associação. A associação entre o grau de sobressaliência e a fase de desenvolvimento da dentição foi estatisticamente significante ( $<<0,001)$. Quanto mais avançada a fase de desenvolvimento da dentição, maior foi a prevalência da sobressaliência exagerada (Tabela 4).

Em relação ao trespasse vertical, a sobremordida exagerada (50 - $27,0 \%$ ) e a mordida aberta ante-

TABELA 1 - Freqüência da forma dos arcos superior e inferior.

\begin{tabular}{c|c|r|r}
\hline \hline \multirow{2}{*}{ Arco } & \multirow{2}{*}{ Forma } & \multicolumn{2}{|c}{ Freqüência } \\
\cline { 3 - 4 } & & $\mathrm{n}$ & $\%$ \\
\hline \multirow{4}{*}{ Superior } & Arredondado & 129 & 68,9 \\
\cline { 2 - 4 } & Triangular & 59 & 31,4 \\
\cline { 2 - 4 } & Total & 188 & 100 \\
\hline \multirow{3}{*}{ Inferior } & Forma de "U”" & 173 & 92 \\
\cline { 2 - 4 } & Quadrado & 15 & 8 \\
\cline { 2 - 4 } & Total & 188 & 100 \\
\hline \hline
\end{tabular}


Castro LA de, Modesto A, Vianna R, Soviero VLM. Estudo transversal da evolução da dentição decídua: forma dos arcos, sobressaliência e sobremordida. Pesqui Odontol Bras 2002;16(4):367-373.

TABELA 2 - Associação entre a forma dos arcos superior e inferior e a fase de desenvolvimento da dentição decídua.

\begin{tabular}{|c|c|c|c|c|c|c|c|c|c|c|c|}
\hline \multirow{2}{*}{ Arco } & \multirow{2}{*}{ Forma } & \multicolumn{8}{|c|}{ Grupos } & \multirow{2}{*}{ Valor de $\mathrm{p}$} & \multirow{2}{*}{$\mathrm{n}$} \\
\hline & & $\mathrm{n}$ & $\%$ & $\mathrm{n}$ & $\%$ & $\mathrm{n}$ & $\%$ & $\mathrm{n}$ & $\%$ & & \\
\hline \multirow{2}{*}{ Superior } & Arredondada & 19 & 100 & 25 & 89,3 & 52 & 63,4 & 30 & 53,6 & \multirow{2}{*}{$<0,001$} & \multirow{2}{*}{$185^{*}$} \\
\hline & Triangular & 0 & 0 & 3 & 10,7 & 30 & 36,6 & 26 & 46,4 & & \\
\hline Inferior & Quadrada & 1 & 5,3 & 0 & 0 & 9 & 11 & 5 & 8,9 & $>0,05$ & $185^{*}$ \\
\hline
\end{tabular}

*3 crianças foram excluídas das análises de associação pois estavam com os incisivos em erupção, não pertencendo, deste modo, a nenhum grupo classificado neste estudo.

TABELA 3 - Freqüência da sobressaliência.

\begin{tabular}{l|c|c}
\hline \multicolumn{1}{c|}{ Sobressaliência } & Freqüência $(\mathrm{n})$ & Percentual $(\%)$ \\
\hline Topo-a-topo & 21 & 11,2 \\
\hline Leve & 57 & 30,3 \\
\hline Moderada & 72 & 38,3 \\
\hline Exagerada & 34 & 18,1 \\
\hline Negativa & 4 & 2,1 \\
\hline Total & 188 & 100 \\
\hline \hline
\end{tabular}

rior (48 - 25,9\%) foram as condições mais prevalentes, seguidas da leve (39-21,1\%) (Tabela 5). A associação entre o grau de sobremordida e a fase de desenvolvimento da dentição foi estatisticamente significante $(\mathrm{p}<0,001)$. Antes da erupção dos dentes, 63,2\% (12) das crianças apresentavam sobremordida topo-a-topo. No Grupo 1, a maior prevalência foi da sobremordida exagerada, $60,7 \%$ (17); no Grupo 2, a distribuição foi uniforme nos vários graus de sobremordida. O Grupo 3 apresentou prevalência mais alta de mordida aberta anterior $(24-42,9 \%)$ (Tabela 6).

\section{DISCUSSÃO}

O gênero não apresentou associação com nenhuma das variáveis estudadas: forma dos arcos dentários, sobressaliência nem sobremordida. No estudo epidemiológico realizado por Tollara ${ }^{20}(2001)$ em que foram verificadas maloclusões em 413 crianças de 6 a 35 meses de idade, o gênero também não esteve associado à sobressaliência e sobremordida exagerada.

A maior prevalência encontrada para a forma do arco superior foi para o arco arredondado, e para o arco inferior a forma em "U", concordando com vários autores que as consideram mais preva- lentes e adequadas para o bom alinhamento dos dentes e para a oclusão $0^{4,15,22}$. As formas dos arcos dentários são consideradas inadequadas para um bom relacionamento entre as bases ósseas quando se apresentam triangular no arco superior e quadrada no arco inferior. Na associação com as fases de desenvolvimento da dentição decídua, constatou-se um aumento da prevalência da forma triangular no arco superior nas fases mais avançadas de desenvolvimento da dentição. Embora não tenha sido avaliada neste estudo, acredita-se que a etiologia desta alteração na forma do arco, encontra-se nos hábitos de sucção nutritivos e não-nutritivos. O prolongamento destes hábitos pode causar os primeiros desvios na forma e nas funções bucais, tornando-se evidentes apenas com o passar do tempo, portanto, em fases mais avançadas da dentição decídua ${ }^{4,5,11,18}$.

Em relação à prevalência da sobressaliência moderada e leve na dentição decídua, os resultados encontrados aqui mostraram concordância com vários autores ${ }^{2,19,20,21}$. Valente, Mussolino ${ }^{21}$ (1989), estudando crianças entre 2 e 4 anos de idade, verificaram a sobressaliência moderada ( 2 a $4 \mathrm{~mm})$ como a mais freqüente $(48,33 \%)$, concordando com os resultados encontrados neste estudo $(38,3 \%)$. Porém, para esses autores, a sobressaliência leve aumentou com a idade, enquanto no presente estudo houve um aumento da sobressaliência exagerada com a idade. Esse aumento da sobressalência em relação à fase mais avançada de desenvolvimento da dentição decídua também foi observado por Tollara ${ }^{20}(2001)$ ao estudar crianças da mesma faixa etária. Ainda é importante considerar que no estudo de Valente, Mussolino ${ }^{21}$ (1989) foram avaliadas crianças que já apresentavam a dentição decídua completa, diferentemente do presente estudo e do de Tollara $^{20}$ (2001), nos quais 
Castro LA de, Modesto A, Vianna R, Soviero VLM. Estudo transversal da evolução da dentição decídua: forma dos arcos, sobressaliência e sobremordida. Pesqui Odontol Bras 2002;16(4):367-373.

TABELA 4 - Associação entre o grau de sobressaliência e a fase de desenvolvimento da dentição decídua.

\begin{tabular}{|c|c|c|c|c|c|c|c|c|}
\hline \multirow{3}{*}{$\begin{array}{c}\text { Grau de } \\
\text { sobressaliência }\end{array}$} & \multicolumn{8}{|c|}{ Grupos } \\
\hline & \multicolumn{2}{|c|}{ Grupo 0} & \multicolumn{2}{|c|}{ Grupo 1} & \multicolumn{2}{|c|}{ Grupo 2} & \multicolumn{2}{|c|}{ Grupo 3} \\
\hline & $\mathrm{n}$ & $\%$ & $\mathrm{n}$ & $\%$ & $\mathrm{n}$ & $\%$ & $\mathrm{n}$ & $\%$ \\
\hline Topo-a-topo & 13 & 68,4 & 2 & 7,4 & 2 & 2,5 & 1 & 1,8 \\
\hline Leve & 2 & 10,5 & 12 & 44,4 & 30 & 3,5 & 13 & 23,6 \\
\hline Moderada & 3 & 15,8 & 11 & 40,7 & 33 & 41,3 & 25 & 45,5 \\
\hline Exagerada & 1 & 5,3 & 2 & 7,4 & 15 & 18,8 & 16 & 29,1 \\
\hline Total & 19 & 100 & 27 & 100 & 80 & 100 & 55 & 100 \\
\hline
\end{tabular}

p < 0,001. Total da amostra: 181 crianças - 3 crianças foram excluídas das análises de associação pois estavam com os incisivos em erupção, não pertencendo, deste modo, a nenhum grupo classificado neste estudo. Nesta associação foram excluídas também 4 crianças que apresentavam sobressaliência negativa.

TABELA 5 - Freqüência da sobremordida.

\begin{tabular}{l|c|c}
\hline \hline \multicolumn{1}{c|}{ Sobremordida } & Freqüência $(\mathrm{n})$ & Percentual (\%) \\
\hline Topo-a-topo & 15 & 8,1 \\
\hline Leve & 39 & 21,1 \\
\hline Moderada & 33 & 17,8 \\
\hline Exagerada & 50 & 27 \\
\hline Negativa & 48 & 25,9 \\
\hline Total & 185 & 100 \\
\hline \hline
\end{tabular}

esta característica foi avaliada ao longo do desenvolvimento da dentição decídua.

A prevalência da sobremordida variou de acordo com a fase de desenvolvimento da dentição decídua. Antes da erupção dos incisivos, a condição mais freqüentemente observada foi a relação topo-a-topo. Após a formação da guia incisal, houve um súbito aumento da sobremordida, porém quando os primeiros molares decíduos erupcionaram, formando o primeiro pilar de oclusão, a sobremordida diminuiu, podendo-se verificar, então, uma distribuição equilibrada entre os diferentes graus de sobremordida. Este comportamento da sobremordida constitui uma característica fisiológica do desenvolvimento da dentição decídua, concordando com vários autores que estudaram a dentição decídua ${ }^{2,11,15,16}$. No entanto, na fase de dentição decídua completa (Grupo 3), houve um significante aumento da mordida aberta anterior. Os resultados encontrados no presente estudo concordam com o estudo de Tollara ${ }^{20}$ (2001), em que foi observado um aumento da mordida aberta anterior nas fases mais avançadas de desenvolvimento da dentição decídua. Outros estudos reali- zados em crianças brasileiras na dentição decídua também encontraram resultados semelhantes em relação à prevalência da sobressaliência moderada e sobremordida negativa ${ }^{19,20,21}$.

Os dados obtidos no presente estudo são muito interessantes ao serem analisados, visto que evidenciam alterações na evolução da dentição decídua, justamente quando os efeitos de possiveis hábitos bucais deléterios tiveram tempo suficiente para se expressarem. Então, pode-se sugerir que o desenvolvimento da sobremordida, ao apresentar um aumento da mordida aberta anterior na fase de dentição decídua completa, esteja condicionado ao tempo necessário para o surgimento dos desvios causados pelos hábitos de sucção, semelhante ao que ocorre com o aparecimento da forma alterada dos arcos dentários decíduos e o desenvolvimento da sobressaliência exagerada na fase de dentição decídua completa.

Segundo Leighton ${ }^{11}$ (1969), a sobremordida pode ser bastante influenciada pelos hábitos bucais, o que coincide com os resultados obtidos no estudo de Serra-Negra et al. ${ }^{18}$ (1997). Os autores concluíram que as crianças que apresentam hábitos bucais deletérios possuem muito mais risco de desenvolverem mordida aberta anterior do que as sem hábitos bucais.

Para Valente, Mussolino ${ }^{21}$ (1989), houve uma diminuição da mordida aberta anterior com a idade, diferente dos resultados obtidos no presente estudo, onde se observou um aumento da freqüência da sobremordida negativa com o passar do tempo. Porém, as faixas etárias nestes dois estudos (de 2 a 6 anos e de 6 a 39 meses de idade, respectivamente) coincidiram apenas parcialmente. 
Castro LA de, Modesto A, Vianna R, Soviero VLM. Estudo transversal da evolução da dentição decídua: forma dos arcos, sobressaliência e sobremordida. Pesqui Odontol Bras 2002;16(4):367-373.

TABELA 6 - Associação entre o grau de sobremordida e a fase de desenvolvimento da dentição decídua.

\begin{tabular}{|c|c|c|c|c|c|c|c|c|}
\hline \multirow{3}{*}{ Sobremordida } & \multicolumn{8}{|c|}{ Grupos } \\
\hline & \multicolumn{2}{|c|}{ Grupo 0} & \multicolumn{2}{|c|}{ Grupo 1} & \multicolumn{2}{|c|}{ Grupo 2} & \multicolumn{2}{|c|}{ Grupo 3} \\
\hline & $\mathrm{n}$ & $\%$ & $\mathrm{n}$ & $\%$ & $\mathrm{n}$ & $\%$ & $\mathrm{n}$ & $\%$ \\
\hline Topo-a-topo & 12 & 3,2 & 2 & 7,1 & 0 & 0 & 1 & 1,8 \\
\hline Leve & 2 & 10,5 & 6 & 21,4 & 20 & 24,4 & 11 & 19,6 \\
\hline Moderada & 1 & 5,3 & 3 & 10,7 & 20 & 24,4 & 9 & 16,1 \\
\hline Exagerada & 0 & 0 & 17 & 60,7 & 22 & 26,8 & 11 & 19,6 \\
\hline Negativa & 4 & 21,1 & 0 & 0 & 20 & 24,4 & 24 & 42,9 \\
\hline Total & 19 & 100 & 28 & 100 & 82 & 100 & 56 & 100 \\
\hline
\end{tabular}

$\mathrm{p}<0,001$. Total da amostra: 185 crianças - 3 crianças foram excluídas das análises de associação pois estavam com os incisivos em erupção, não pertencendo, deste modo, a nenhum grupo classificado neste estudo.

Neste estudo, pudemos observar o aparecimento de algumas importantes características da dentição decídua, o que possibilitou, oportunamente, o acompanhamento das rápidas modificações que ocorrem durante a evolução da dentição decídua. Assim, foi possivel verificar que os sinais incipientes de maloclusões surgem com a evolução da dentição decídua, o que corrobora com outros estudos sobre a prevalência de maloclusões em idades bastante jovens ${ }^{6,18,19,20}$. Dessa forma, torna-se extremamente importante que a primeira visita odontológica ocorra no primeiro ano de vida, possibilitando ao odontopediatra a prevenção ou diagnóstico precoce de maloclusões na dentição decídua em desenvolvimento.

\section{REFERÊNCIAS}

1. Ando T, Psillakis CM. Considerações sobre rebordos gengivais do recém-nascido. Rev Fac Odontol Univ São Paulo 1973;11(1):155-62.

2. Baume LJ. Physiological tooth migration and its significance for the development of occlusion. IV - the biogenesis of overbite. J Dent Res 1950;29(4):440-7.

3. Bishara SE, Nowak AJ, Kohout FJ, Heckert DA, Hogan M. Influence of feeding and non-nutritive sucking methods on the development of the dental arches: longitudinal study of the first 18 months of life. Pediatr Dent 1987;9(1):13-21.

4. Camargo MCF. Programa preventivo de maloclusões em bebês. In: Gonçalves EAN, Feller C. Atualização na Clínica Odontológica. São Paulo: Artes Médicas; 1998. p.405-42.

5. Farsi NM, Salama FS. Sucking habits in Saudi children: prevalence, contributing factors and effects on the primary dentition. Pediatr Dent 1997;19(1):28-33.

6. Foster DO, Hamilton MC. Occlusion in the primary dentition: study of children at $21 / 2$ to 3 years of age. Br Dent $J$ 1969;126(2):76-9.

\section{CONCLUSÃO}

Não houve diferença estatística das diversas formas de arcos dentários, da sobressaliência e da sobremordida em relação ao gênero da criança.

A forma das arcadas mais freqüentes foram: no arco superior, a forma arredondada, e, no arco inferior, a forma em "U", consideradas as mais adequadas.

A fase de desenvolvimento da dentição decídua influenciou na forma dos arcos superiores, no grau da sobressaliência e da sobremordida.

As maloclusões mais encontradas nas fases mais avançadas de desenvolvimento da dentição decídua foram: a sobressaliência exagerada e a mordida aberta anterior.

7. Graber TM, Vanarsdall Júnior RL, Barbosa JA. Ortodontia princípios e técnicas atuais. $2^{a}$ ed. Rio de Janeiro: Guanabara Koogan; 1996. p.897.

8. Haddad AE, Corrêa MSNP. Desenvolvimento das funções bucais no bebê. In: Corrêa MSNP. Odontopediatria na primeira infância. São Paulo: Santos; 1998, p.55-63.

9. Infante PF. Sex differencs in the chronology of deciduous tooth emergence in white and black children. J Dent Res 1974;53(2):418-21.

10. Legovig M, Ostric L. The effects of feeding methods on the growth of the jaws in infants. ASDC $J$ Dent Child 1991;58(3):253-5.

11. Leigthon BC. The early signs of maloclusion. Trans Europ Orthod Soc 1969;6(1):353-63.

12. Moss ML. The functional matrix hypothesis revisited.1 the role of mechanotransduction. Am J Orthod DentoFacial Orthop 1997;112(1):8-11.

13. Moss ML. The functional matrix hypothesis revisited. 2 the pole of an osseous connected cellular network. Am J Orthod DentoFacial Orthop 1997;112(2):221-6. 
Castro LA de, Modesto A, Vianna R, Soviero VLM. Estudo transversal da evolução da dentição decídua: forma dos arcos, sobressaliência e sobremordida. Pesqui Odontol Bras 2002;16(4):367-373.

14. Moss ML. The functional matrix hypothesis revisited. $3-$ the genomic thesis. Am J Orthod DentoFacial Orthop 1997; 112(3):338-42.

15. Moyers RE. Ortodontia. $4^{\mathrm{a}}$ ed. Rio de Janeiro: Guanabara Koogan; 1991.

16. Proffit WR, Fields HWJ. Ortodontia contemporânea. $2^{\mathrm{a}}$ ed. Rio de Janeiro: Guanabara Koogan; 1995.

17. Sanchez ALSF. Contribuição ao estudo das características das arcadas de recém-nascidos. [Dissertação de Mestrado]. Rio de Janeiro: Faculdade de Odontologia da Universidade Federal do Rio de Janeiro; 2000.

18. Serra-Negra JMC, Pordeus IA, Rocha Júnior JF. Estudo da associação entre aleitamento, hábitos bucais e maloclusões. Rev Odontol Univ São Paulo 1997;11(2):79-86.

19. Soviero VLVM. Estudo da influência do tipo e duração do aleitamento no desenvolvimento de hábitos de sucção não nutritivos e no relacionamento entre as arcadas decíduas. [Tese de Doutorado]. Rio de Janeiro: Faculdade de Odontologia da UFRJ; 1999.

20. Tollara MCRN. Estudo epidemiológico da prevalência de maloclusão em crianças de 5 a 35 meses de idade no município de Diadema - São Paulo, Brasil. [Dissertação de Mestrado]. São Paulo: Faculdade de Odontologia da USP; 2001.

21. Valente A, Mussolino ZM. Freqüência de sobressaliência, sobremordida e mordida aberta na dentição decídua. Rev Odontol Univ São Paulo 1989;3(3):402-7.

22. Villena RS, Corrêa MSNP. Características do sistema estomatognático: algumas anomalias no recém-nascido. In: Corrêa MSNP. Odontopediatria na primeira infância. São Paulo: Santos; 1998 p.87-90.

Recebido para publicação em 06/11/01

Enviado para reformulação em 29/04/02

Aceito para publicação em 20/08/02 